Gdańsk 2020, Nr. 43

https://doi.org/10.26881/sgg.2020.43.09

Magdalena Pieklarz-Thien

Universität Olsztyn / Uniwersytet Warmińsko-Mazurski w Olsztynie

ORCID: 0000-0002-9153-431X

\title{
Zum Ertrag der germanistischen Sprachwissenschaft für die DaF-Didaktik in Polen: Der sprachliche Lerngegenstand gesprochenes Standarddeutsch
}

\begin{abstract}
Sprache ist ein facettenreicher Gegenstand, mit dem sich eine Reihe von wissenschaftlichen Disziplinen unter verschiedenen Blickwinkeln beschäftigt. Es ist vor allem die Sprachwissenschaft, welche die Sprache als solche zum Objekt ihrer Untersuchungen und Überlegungen macht und zweifelsohne als eine Expertendisziplin verstanden wird. Interesse am Gegenstand Sprache haben allerdings auch andere Disziplinen (wie z. B. die Sprachdidaktik), auch wenn sie unter einer anderen Perspektive und aus einer anderen Motivation heraus arbeiten und damit auf andere Arbeitslogiken, Begrifflichkeiten und Referenzsysteme zurückgreifen. Das Ziel dieses Beitrags besteht darin, über den Ertrag der germanistischen Sprachwissenschaft für die DaF-Didaktik in Polen nachzudenken. Die Überlegungen werden am Beispiel des gesprochenen Standarddeutsch als Lehr- und Lerngegenstand angestellt.
\end{abstract}

Schlüsselwörter: Sprache, Sprachwissenschaft, Sprachdidaktik, gesprochenes Standarddeutsch, germanistische DaF-Didaktik

On the Usefulness of German Linguistics for Teaching German as a Foreign Language in Poland on the Example of a Standard Spoken German. - Language is a multidimensional object of cognition, which is dealt with by many scientific disciplines with different research perspectives. Linguistics is particularly important, as it makes language its primary research subject and is undoubtedly seen as the leading discipline in comparison with other sciences interested in language. However, for them too, language is an important object of cognition in certain cognitive areas, studied and described from the point of view of disciplinarily specific logic, terminology and reference semantics. This article attempts to reflect on the role and usefulness of the results of German linguistics research for teaching German as a foreign language during German studies in Poland. Considerations are illustrated by an example of a standard spoken German as a subject of teaching in philological education in Poland.

Keywords: language, linguistics, foreign language education, standard spoken German, language education at German studies.

\section{Einleitung: grundlegende Annahmen und Zielsetzung des Beitrags}

Bei der Sprachwissenschaft und Sprachdidaktik handelt es sich um zwei wissenschaftliche Disziplinen, die ein gemeinsamer, wenngleich anders perspektivierter, Gegenstand verbindet, 
sowie auch ein sensibles und in der Fachliteratur viel diskutiertes Miteinander charakterisiert. Diese Feststellungen deuten zweifellos auf ein gewisses Konflikt- und Spannungspotenzial hin, das dem gegenseitigen Austausch und der Wahrnehmung gegenseitiger Erkenntnisse im Wege stehen kann. Je stärker allerdings die Beziehungen zwischen den Wissenschaften von Spannungen geprägt sind, umso gewinnbringender sind jegliche disziplinäre Grenzüberschreitungen und Kooperationen, deren Ertrag eine Innovation und Optimierung für ihre Verwendungsfelder bedeuten kann.

Ohne das schwierige Verhältnis der beiden Wissenschaften eingehend zu beleuchten ${ }^{1}$, wird in diesem Beitrag davon ausgegangen, dass die germanistische Sprachwissenschaft über das entscheidende Expertenwissen in Bezug auf die Analyse und Beschreibung von Strukturen und Funktionen sprachlicher Einheiten verfügt. Dieses Wissen stellt sie der DaF-Didaktik, genuin der DaF-Linguistik ${ }^{2}$, bereit, damit diese diverse Aspekte ihrer wissenschaftlich fundierten Bewusstmachung und unterrichtlicher Vermittlung erforschen und beschreiben kann und folglich ihrem Anwendungsfeld, dem DaF-Unterricht, in Form von brauchbaren Konzepten und Ansätzen zur Verfügung stellt. Vor dem Hintergrund dieser Grundannahmen besteht das Ziel des Beitrags darin, über den Ertrag der germanistischen Sprachwissenschaft für die DaF-Didaktik in Polen nachzudenken, wobei aus der Vielfalt der möglichen sprachlichen Gegenstände gesprochenes Standarddeutsch als ein anspruchsvolles Lehr- und Lernobjekt im germanistischen DaF-Unterricht im Ausland ausgewählt wird. Da bei einer so skizzierten Zielsetzung konzeptuelle Diskussionen wichtig und fruchtbar sein können, werden in einem ersten Schritt Überlegungen zu den relevanten disziplinären Zuständigkeiten und Anwendungsfeldern für gesprochenes Standarddeutsch kurz dargestellt (vgl. 2). In einem zweiten Schritt werden diesbezügliche relevante Arbeiten der Germanistischen Sprachwissenschaft aufgelistet (vgl. 3.1), die von polnischen Sprachforschern und Sprachdidaktikern rezipiert wurden und zur Weiterentwicklung der Reflexion in der polnischen DaF-Didaktik/-Linguistik beigetragen haben (vgl. 3.2).

\section{Gesprochenes Standarddeutsch im Spannungsverhältnis von Sprachwissenschaft, Sprachdidaktik und Sprachunterricht}

Es ist selbstverständlich, dass sprachliche Lerngegenstände in Bezug auf ihre inhärente Komplexität, ihre didaktischen Aufbereitungspotenziale sowie die Schwierigkeit ihres Erlernens im institutionellen Fremdsprachenunterricht im Ausland sehr unterschiedlich sind. Weniger klar ist allerdings, welche Attribute und internen Prozesse der für sie zuständigen Disziplinen manche linguistischen Felder der Sprachbeschreibung zu komplexen, didaktisch problematischen und im Unterricht vernachlässigten Lerngegenständen machen. Sich Klarheit über

\footnotetext{
1 Dies ist in Pieklarz-Thien (2018a) und (2020a) getan.

2 Unter der DaF-Linguistik verstehe ich mit Fandrych (62016: 36, 2010: 173) eine Subdisziplin und Forschungslandschaft der DaF-Didaktik, welche die deutsche Sprache aus der Perspektive der Lernenden, die Deutsch als weitere Sprache auf der Folie mindestens einer anderen Muttersprache erwerben, betrachtet, untersucht und beschreibt.
} 
die disziplinären Zuständigkeiten zu verschaffen, kann als eine elementare Überlegung zur Erschließung und umfassenden didaktischen Perspektivierung der Erkenntnisobjekte verstanden werden.

Es unterliegt keinem Zweifel, dass die Germanistische Sprachwissenschaft diejenige Disziplin ist, welche der DaF-Linguistik brauchbare und homogene Erkenntnisse in Bezug auf die sprachlichen Gegenstände zur Verfügung stellen kann. Auch wenn die Trennung zwischen Germanistischer Sprachwissenschaft und $\mathrm{DaF}$ inhaltlich, institutionell und personell nicht immer ganz exakt zu ziehen ist (Thurmair 2018: 409), setzen entsprechende Analysen und Forschungen in der Germanistischen Sprachwissenschaft didaktische Operationalisierungen sprachlicher Gegenstände in der DaF-Linguistik voraus. Wenn in der Germanistischen Linguistik entsprechende Erkenntnisse nicht gewonnen werden, bzw. diese nicht homogen sind, d. h. kontroverse und diskussionsbedürftige Thesen beinhalten, fehlt der DaF-Linguistik eine Basis für ihre Forschung und Entwicklung der didaktischen Ansätze. Unumstritten dabei ist, dass es nicht reicht, sprachwissenschaftliche Vorannahmen und Modellierungen ungebrochen an den DaF-Unterricht zu übertragen. Die Aufgabe der DaF-Linguistik ist es, in ihren Forschungen klar herauszustellen, wie sprachliche Strukturen und Funktionen im Unterricht bearbeitet werden sollten, damit sie dann in spontanen mündlichen und schriftlichen Äußerungen der Lernenden präsent sind. Wichtig ist dabei, die Dynamik und Instabilität des linguistischen (wie jedes anderen wissenschaftlichen) Wissens nicht zu unterschätzen, das Strömungen und Moden unterworfen und durch das ständige Hinterfragen von Prämissen, Methoden und Resultaten geprägt ist (Berthele 2009: 10). Die DaF-Didaktik/-Linguistik sollte sich daher, wie Albert (1995: 82) es bereits in den 1990er Jahren zugespitzt formulierte, nicht „an jede Mode in der Linguistik anhängen“. Nicht alle Erkenntnisse, Methoden und Betrachtungsweisen der Germanistischen Linguistik sind im gleichen Maße prädestiniert, Relevantes für den DaF-Unterricht abzuwerfen. Es kann sich also nur um Adaptation und Umsetzung derjenigen linguistischen Erkenntnisse und Betrachtungen handeln, die die Theorie und Praxis des DaF-Unterrichts fundieren, bereichern und optimieren können.

Vor diesem Hintergrund lässt sich auch gesprochenes Standarddeutsch als sprachlicher Lehr- und Lerngegenstand disziplinär verorten. Die Abbildung 1 stellt es im Spannungsverhältnis von zuständigen Disziplinen und Anwendungsfeldern dar.

Eine solche wissenschaftliche Verortung ist insofern relevant, als viele Begriffe und Objekte in der Alltagssprache und Alltagspraxis vorwissenschaftlich gegeben sind. Dies gilt zweifellos auch für die Fremdsprachendidaktik als Wissenschaft, die mit verschiedenen Typen von Evidenz arbeitet, die von den jeweiligen zugrundeliegenden plausibilitätsdidaktischen / theoretischen, empirischen oder linguistischen Ansätzen abhängig sind (Rothstein u. a. 2014: 204). Auch wenn immer wieder für ein Zusammenspiel aller drei Ansätze plädiert wird, zeigt die Forschungspraxis der gegenwärtigen Sprachdidaktik allgemein und der DaF-Didaktik speziell, dass ein Wechselspiel aller drei Forschungsrichtungen selten der Fall ist. So findet man in der DaF-Didaktik zahlreiche erfahrungsbasierte, durch Subjektivität geprägte und auf plausiblen Annahmen operierende Ansätze, die linguistisch problematisch erscheinen und auch empirisch nicht überprüft worden sind. Diese aufzudecken und folglich entsprechende neue, linguistisch fundierte Wege und Alternativen aufzuzeigen, ist eine der Hauptaufgaben der DaF-Linguistik. 


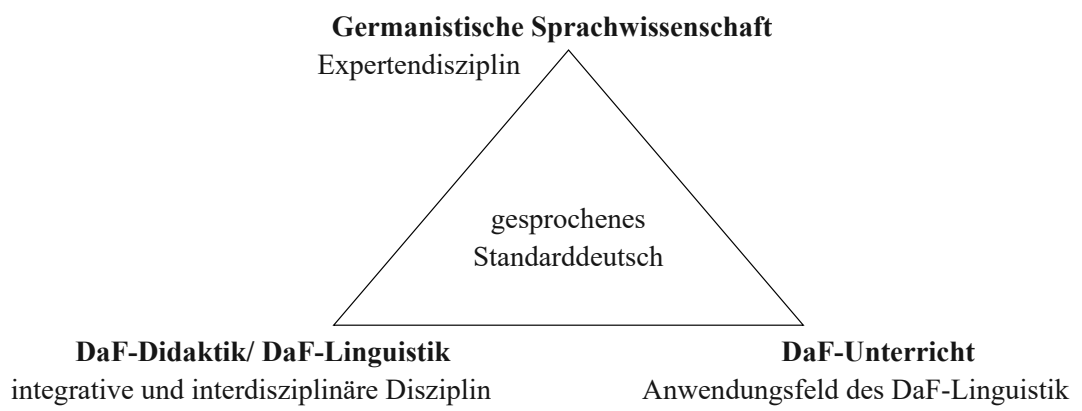

Abb. 1: Gesprochenes Standarddeutsch als sprachlicher Lehr- und Lerngegenstand im Spannungsverhältnis von Disziplinen und Anwendungsfeldern

\section{Forschungsüberblick zum gesprochenen Standarddeutsch in sprachdidaktischer Perspektive}

Im Zusammenhang mit der seit den 1960er Jahren intensiv betriebenen empirischen Untersuchung des realen Sprachgebrauchs werden zahlreiche Erkenntnisse gewonnen, die Divergenzen bzw. Auseinanderentwicklungen zwischen der Erforschung der Gegenwartssprache, wie sie von muttersprachlichen Sprechern verwendet wird, und der systemlinguistischen Vermittlung von Wissen über Sprache und der Förderung sprachlicher Kompetenzen im DaF-Unterricht belegen. So mehren sich im In- und Ausland verstärkt Stimmen, welche die hauptsächliche Ausrichtung des DaF-Unterrichts an der normierten Schriftsprache bezweifeln und die Fachgemeinschaft dazu auffordern, zu überlegen, welche Normen und Varietäten der deutschen Sprache in die unterschiedlichen Vermittlungskontexte einzubeziehen sind. Die meiste Aufmerksamkeit der Fachdiskussion gilt dabei der Vermittlung der gesprochenen Sprache, was angesichts der Tatsache, dass diese Varietät „im Vergleich zu formeller Schriftsprache ontound phylogenetisch primär ist, kognitiv geringere Anforderungen stellt, in der Gesellschaft den kommunikativen Nähebereich abdeckt und damit auch quantitativ bei der Masse der Bevölkerung den größeren Kommunikationsraum ausfüllt" (Elspaß 2010: 420), nicht verwunderlich ist. Die Notwendigkeit ihrer Vermittlung liegt aber auch darin, dass sie die wichtigsten Sinnverleihungen beinhaltet sowie elementare Denkstrukturen und Wahrnehmungen der Welt verfestigt, sodass ihre Beherrschung die Teilhabe an der fremdsprachlichen Kultur ermöglichen kann (Bartmiński 2010: 116-117).

Im Folgenden werden Arbeiten der Germanistischen Sprachwissenschaft genannt, welche gesprochenes Standarddeutsch ${ }^{3}$ vor allem als ein fremdsprachendidaktisches Erkenntnisobjekt betrachten und perspektivieren. Ebenfalls voranzustellen ist, dass man in der

3 Unter gesprochenem Standarddeutsch verstehe ich eine überregionale, mündliche Varietät der deutschen Sprache mit ihren spezifischen, vom schriftlichen Standard abweichenden Gebrauchsnormen, die in alltags- und nähesprachlichen Interaktionen verwendet werden (Schneider / Butterworth / Hahn 2018). Auf 
deutschsprachigen Fachliteratur der letzten fünfzig Jahre für diesen Gegenstandsbereich mehrere Bezeichnungen wie gesprochene Sprache, gesprochenes Deutsch, Alltagssprache, Interaktionale Sprache, gesprochener Standard, gesprochenes Standarddeutsch benutzt, die aus der sprachdidaktischen Perspektive semantisch einander sehr ähnlich sind. Auch wenn die Sprachforschung die semantische Füllung des Gegenstandsbereichs in Bezug auf unterschiedliche linguistische, soziologische, gesprächs- und kommunikationstheoretische Ansätze immer wieder diskutiert, scheint es aus sprachdidaktischer Perspektive nicht sinnvoll zu sein, alle diese Termini für den DaF-Unterricht zu operationalisieren. Genauso unfruchtbar wäre es, eine dogmatische disziplinäre Zuordnung der Forschenden und ihrer Arbeiten vorzunehmen, welche die Grenzüberschreitungen und Interdisziplinarität der Wissenschaften wie auch die Komplexität und Multidimensionalität der Erkenntnisobjekte nicht berücksichtigt.

3.1 Arbeiten der didaktisch interessierten Germanistischen Sprachwissenschaft im deutschsprachigen Raum und international

Im Bereich der fremdsprachenunterrichtlichen Belange liegen für den Gegenstand gesprochenes Standarddeutsch inzwischen relativ viele theoretische Überlegungen wie auch einige Umsetzungsvorschläge vor, die allerdings noch nicht als eine ganzheitliche, kontinuierliche und systematische Erforschung und Erfassung des Gegenstandes in didaktischer Perspektive gesehen werden können. Ein Grund dafür liegt sicherlich in der Komplexität und der unzureichenden Erforschung des Gegenstandes, welche einige Theoriedefizite (Ágel/Hennig 2007: VII) zur Folge haben. Diese äußern sich u. a. darin, dass kein Konsens über die Integration der Ergebnisse der Gesprochene-Sprache-Forschung in traditionelle Grammatiken besteht, so dass man nur bedingt Konzepte ihrer Vermittlung im DaF-Unterricht entwickeln kann.

Nichtsdestotrotz hat in den letzten drei Jahrzehnten die Gesprochene-Sprache-Forschung, aber auch die Angewandte Gesprächsforschung und neulich auch die Interaktionale Linguistik eine ganze Reihe von Erkenntnissen gewonnen, die für die Sprachdidaktik ${ }^{4}$ eine unmittelbare Anwendungsrelevanz haben. So sind einerseits zahlreiche theoretische, didaktisch motivierte Beiträge entstanden, die sich grundlegend mit Möglichkeiten und Herausforderungen der Vermittlung des gesprochenen Deutsch im DaF-Unterricht beschäftigen, andererseits auch einige Einzelstudien zu ausgewählten Eigenschaften des gesprochenen Deutsch, die auch konkrete Didaktisierungsvorschläge mit dem dazugehörigen Übungsmaterial beinhalten. Die Weiterentwicklung der didaktischen Reflexion in diesem Bereich unterstützen zweifellos die entstandenen Referenzwerke wie Einführungen (Schwitalla 1997, Henning 2006, Imo / Lanwer 2019) und einige Grammatiken $^{5}$, die zum Teil auch gesprochensprachliche und interaktionale Sprachmuster und -normen

die in der Sprachforschung intensiv geführte Diskussion über die Normativität und Standardisierung des Deutschen und diesbezüglich berechtigten Bedenken kann ich an dieser Stelle nicht eingehen.

4 Dies gilt sowohl für die Didaktik des Deutschen als Muttersprache, Zweitsprache als auch für die im Folgenden fokussierte Didaktik des Deutschen der Fremdsprache.

5 Die Relevanz der Grammatiken und linguistischen Einführungen ergibt sich daraus, dass ihnen „eine Mittlerfunktion zwischen linguistischer Forschung und Praxis des Deutschen als Fremdsprache zukommt“ (Hennig 2001: 5). 
thematisieren wie die Grammatik der deutschen Sprache von Zifonun et al. (1997) oder die Textgrammatik der deutschen Sprache von Weinrich ('2007) und nicht zuletzt dieDuden-Grammatik, die seit 2005 ein umfassendes Kapitel zur gesprochenen Sprache von Reinhard Fiehler beinhaltet.

Es ist sicherlich nicht möglich, alle Forschenden, alle Arbeiten wie auch alle Diskussionsstränge der letzten dreißig Jahre zum gesprochenen Standarddeutsch als Lehr- und Lerngegenstand im DaF-Unterricht zu nennen. Besonders ertragreich sind $\mathrm{m}$. E. Untersuchungen zur Gesprächskompetenz, der sprachlichen Höflichkeit, Indirektheit und Natürlichkeit von Lehrbuchdialogen von Heinz-Helmut Lüger (1991, 1993, 1995a, 1995b, 2009), die eine lebhafte Diskussion um den Gegenstand Gespräch im Lehrwerk und dort insbesondere die Angemessenheit der sprachlichen Mittel und Strukturen in den Lehrwerksdialogen angestoßen haben, die auch weiter nach der Jahrtausendwende geführt wird (Vorderwülbecke 2008, Al-Nasser 2011, Günthner / Wegner / Weidner 2013). Auch die korpusbasierten Analysen zu einzelnen Phänomenen des mündlichen überregionalen Sprachgebrauchs von Susanne Günthner (u. a. 2000a, 2000b, 2002a, 2002b, 2008, 2010, 2011a, 2011b) und ihren Mitarbeitern und Forschungspartnern, darunter besonders von Wolfgang Imo (2008, 2009, 201 1, 2012, 2013a, 2013b, 2015), haben neue linguistisch fundierte Perspektiven und Relativierungen in dieses Themenfeld gebracht und damit auf die Notwendigkeit einer stärkeren Vernetzung zwischen Sprachforschung und Sprachdidaktik hingewiesen. Aus der Perspektive der linguistisch motivierten Sprachdidaktik und vor allem des muttersprachlichen Deutschunterrichts beleuchtet den Gegenstand Eva Neuland (2006, 2011, 2013, 2018), wobei sie ihn übergreifend vor dem Hintergrund der anderen unterrichtsrelevanten Themen wie der Sprachvariation, des Sprachwandels und der Sprachnormproblematik diskutiert. Linguistisch und didaktisch relevante Impulse liefern verstärkt ebenfalls Vertreter mehrerer Auslandsgermanistiken wie Martin Durell in England (1995, 2004, 2006), Sandro Moraldo in Italien $(2012,2013)^{6}$, Bernd Sieberg in Portugal (2013), die Belange der jeweiligen Germanistiken und ihrer Studierenden berücksichtigen. Darüber hinaus lassen sich viele einzelne Beiträge nennen, deren Autoren sich mit grundlegenden Fragen der Vermittlung vom gesprochenen Deutsch befassen wie Thurmair (2002, 2005, 2013, 2018), Breindl / Thurmair (2003), Schilling / Stezano Cotelo (2008), Bachmann-Stein (2013), Reershemius (1998), Richter (2002), Kilian (2005), Spiekermann (2007) etc. Nicht zuletzt sind zwei aktuelle Werke zu nennen, eine Monographie (Schneider / Butterworth / Hahn 2018) und ein Sammelband (Albert / DiaoKlaeger 2018), die ein gewinnbringender Ertrag eines DFG-Projektes zur Ermittlung des gesprochenen Standards sind und sicherlich von der DaF-Didaktik wahrgenommen werden.

\subsection{Arbeiten der didaktisch interessierten Germanistischen Sprachwissenschaft und der DaF-Didaktik in Polen}

In Polen hat sich vor allem die Warschauer Germanistin Ewa Tomczyk-Popińska (1994, 1997, 2003, 2006) mit dem Gegenstand schon in den 1990er Jahren beschäftigt. Ihre Forschung umfasste sowohl mediolektale Unterschiede im dialogischen Gegenwartsdeutsch wie auch

6 Auch die Sammelbände von Reeg / Gallo / Moraldo (2012), Moraldo / Missaglia (2013), Imo / Moraldo (2015) dürfen an dieser Stelle nicht fehlen. 
explizit didaktische Fragestellungen und insbesondere die Sprache der Lehrwerk-Dialoge. Darüber hinaus haben sich dem Thema einzelne Kolleginnen wie Prokop (1990), Schatte (1993) und Bilut-Homplewicz (2005) gewidmet, indem sie das gesprochene Deutsch untersucht bzw. die Erwerbsschwierigkeiten des gesprochenen Deutsch bei den polnischen Germanistikstudierenden thematisiert haben. Von Pieklarz-Thien (2015, 2018b, 2020b) liegt zudem ein größerer Versuch vor, die gewonnenen Erkenntnisse der Germanistischen Sprachwissenschaft im Bereich des gesprochenen Standarddeutsch zu bündeln und eine bisher ausstehende sowohl theoretisch als auch empirisch begründete, adressatengerechte und interdisziplinär angelegte Didaktik des gesprochenen Deutsch für die polnische Germanistik zu skizzieren.

Es kann angenommen werden, dass Erkenntnisse der Germanistischen Linguistik in diesem Themenfeld in Polen weiterhin rezipiert und zukünftig auch operationalisiert werden. Allerdings muss in diesem Kontext erwähnt werden, dass zwischen Forschung und Lehre, d. h. zwischen dem Gewinnen von klaren und homogenen Erkenntnissen durch die Sprachforschung und ihrer gewinnbringenden Nutzbarmachung durch die Sprachdidaktik, eine universelle Zeitspanne existiert (Kotin 2019: 47), so dass von einem Gleichschritt beider Disziplinen nicht die Rede sein kann ${ }^{7}$. Die linguistische Erkenntnisse adaptierende und umsetzende Sprachdidaktik sollte sich daher auch nichts vorwerfen lassen, soweit ihre Verspätungen nötig und damit begründet sind, d. h. nicht zu lange andauern. Sprachdidaktik ist, wie Kotin (ebd.) zu Recht ausführt, von Natur aus konservativer als die Sprachforschung und zusätzlich von nicht unwesentlichen Einschränkungen geprägt.

\section{Literatur}

Ágel, Vilmos / Hennig, Mathilde (2007): Einleitung. In: Vilmos Ágel / Mathilde Hennig (Hg.): Zugänge zur Grammatik der gesprochenen Sprache. Tübingen: Niemeyer, VII-XIX.

Albert, Georg / Diao-Klaeger, Sabine (Hg.) (2018): Mündlicher Sprachgebrauch zwischen Normorientierung und pragmatischen Spielräumen. Tübingen: Stauffenburg.

Albert, Ruth (1995): Der Bedarf des Fachs ,Deutsch als Fremdsprache' an linguistischer Forschung. In: $D a F 2,82-90$.

Al-Nasser, Mohammed (2011): Gesprochene Sprache im Deutsch-als-Fremdsprache-Unterricht. Eigenschaften der gesprochenen Sprache in Lehrwerkdialogen, unter: https: //epub.uni-bayreuth.de/336/1/ Gesprochene_Sprache_im_DaF_Unterricht.pdf (15.12.2019).

Bachmann-Stein, Andrea / Stein, Stephan 2009: Mediale Varietäten und Fremdsprachendidaktik - Zur Einführung. In: Andrea Bachmann-Stein / Stephan, Stein (Hg.): Mediale Varietäten: Gesprochene und geschriebene Sprache und ihre fremdsprachendidaktischen Potenziale. Landau: Verlag Empirische Pädagogik, 7-12.

Bartmiński, Jerzy ( ${ }^{32010): ~ S t y l ~ p o t o c z n y ~[U m g a n g s s p r a c h e] . ~ I n: ~ J e r z y ~ B a r t m i n ́ s k i ~(H g .): ~ W s p o ́ t c z e s n y ~}$ jezyk polski [Die polnische Sprache der Gegenwart]. Lublin: Wydawnictwo UMCS, 115-134.

\footnotetext{
Ein geeignetes Beispiel für eine solche Zeitspanne wäre das 1971 erschienene, akademische Lehrwerk „Gesprochenes Deutsch“ von Wawrzyniak. Die präsentierten und zu übenden Strukturen in diesem Buch entsprechen nicht dem heutigen Verständnis vom gesprochenen Standarddeutsch.
} 
Berthele, Raphael (2009): Überlegungen zur quasi totalen aber vollkommen normalen Nutzlosigkeit sprachwissenschaftlicher Forschung für die Unterrichtspraxis. In: $L i L i$ 1, 10-25.

Bilut-Homplewicz, Zofia (2005): Zur Sprachnorm-Auffassung im muttersprachlichen und fremdsprachlichen Kontext. In: Danuta Stanulewicz u. a. (Hg.): De lingua et litteris: Studia in honorem Casimiri Andreae Sroka. Gdańsk: Wyd. Uniwersytetu Gdańskiego, 45-51.

Breindl, Eva / Thurmair, Maria 2003: Wie viele Grammatiken verträgt der Lerner? Zum Stellenwert einer <Grammatik der gesprochenen Sprache> (nicht nur) für Deutsch als Fremdsprache. In: Deutsch als Fremdsprache 40/2, 87-93.

Durrell, Martin (1995): Sprachliche Variation als Kommunikationsbarriere. In: Heidrun Popp (Hg.): Deutsch als Fremdsprache. An den Quellen des Faches. Festschrift für Gerhard Helbig zum 65. Geburtstag. München: Iudicium, 417-428.

Durrell, Martin (2004): Variation im Deutschen aus der Sicht von Deutsch als Fremdsprache. In: Der Deutschunterricht 1, 69-77.

Durrell, Martin (2006): Deutsche Standardsprache und Registervielfalt im DaF-Unterricht. In: Eva Neuland (Hg.): Variation im heutigen Deutsch: Perspektiven für den Sprachunterricht. Frankfurt: Lang, 111-122.

Elspaß, Stephan (2010): Alltagsdeutsch. In: Hans-Jürgen Krumm u. a. (Hg.): Deutsch als Fremd-und Zweitsprache. Berlin etc.: de Gruyter, 418-424.

Fandrych, Christian (2010): Grundlagen der Linguistik im Fach Deutsch als Fremd- und Zweitsprache. In: Hans-Jürgen Krumm u. a. (Hg.): Deutsch als Fremd-und Zweitsprache. Berlin: De Gruyter, 173-188.

Fandrych, Christian ('2016): Fokus: Sprache. In: Eva Burwitz-Melzer u. a. (Hg.): Handbuch Fremdsprachenunterricht. Tübingen: A. Francke, 34-38.

Günthner, Susanne (2000a): Grammatik der gesprochenen Sprache - eine Herausforderung für Deutsch als Fremdsprache? In: Info DaF 27/4, 352-366.

Günthner, Susanne (2000b): Zwischen direkter und indirekter Rede. Formen der Redewiedergabe in Alltagsgesprächen. In: Zeitschrift für Germanistische Linguistik 28/1, 1-22.

Günthner, Susanne (2002a): Konnektoren im gesprochenen Deutsch: Normverstoß oder funktionale Differenzierung? In: Deutsch als Fremdsprache. Zeitschrift zur Theorie und Praxis des Deutschunterrichts für Ausländer 39/2, 67-74.

Günthner, Susanne (2002b): Zum kausalen und konzessiven Gebrauch des Konnektors wo im gesprochenen Umgangsdeutsch. In: Zeitschrift für Germanistische Linguistik 30/3, 310-341.

Günthner, Susanne (2008): , weil - es ist zu spät'. Geht die Nebensatzstellung im Deutschen verloren? In: Markus Denkler u. a. (Hg.): Frischwärts und Unkaputtbar. Sprachverfall oder Sprachwandel im Deutschen? Münster: Aschendorff, 103-128.

Günthner, Susanne (2010): ,ICH (-) die karTOFFeln fertig ${ }^{c}$ - Brauchen wir die ,Gesprochene Sprache' in der Auslandsgermanistik?. In: Rudolf Suntrup u. a. (Hg.): Usbekisch-deutsche Studien III: Sprache - Literatur - Kultur - Didaktik. Münster: LIT- Verlag, 241-280.

Günthner, Susanne (2011a): Übergänge zwischen Standard und Non-Standard - welches Deutsch vermitteln wir im DaF-Unterricht? In: Eva L. Wyss, Daniel Stotz (Hg.): Sprachkompetenz in Ausbildung und Beruf. Übergänge und Transformationen. Neuenburg / Neuchâtel, Bulletin VALS ASLA 94/201, 24-47. 
Günthner, Susanne (2011b): Syntax des gesprochenen Deutsch. In: Sandro Moraldo (Hg.): Deutsch Aktuell 2. Tendenzen der deutschen Gegenwartssprache. Rom: Carocci, 108-126.

Günthner, Susanne / Wegner, Lars / Weidner, Beate (2013): Gesprochene Sprache im DaF-Unterricht - Möglichkeiten der Vernetzung der Gesprochene-Sprache-Forschung mit der Fremdsprachenvermittlung. In: Sandro M. Moraldo, Federica Missaglia (Hg.): Gesprochene Sprache im DaF-Unterricht. Grundlagen - Ansätze - Praxis. Heidelberg: Winter, 113-150.

Hennig, Mathilde (2001): Welche Grammatik braucht der Mensch? Grammatikführer für Deutsch als Fremdsprache. München: iudicium.

Hennig, Mathilde (2006): Grammatik der gesprochenen Sprache in Theorie und Praxis. Kassel: University Press.

Imo, Wolfgang (2008): Wenn mündliche Syntax zum schriftlichen Standard wird: Konsequenzen für den Normbegriff im Deutschunterricht. In: Markus Denkler u. a. (Hg.): frischwärts und unkaputtbar. Sprachwandel oder Sprachverfall im Deutschen? Münster: Aschendorff, 153-180.

Imo, Wolfgang (2009): Welchen Stellenwert sollen und können Ergebnisse der Gesprochenen-SpracheForschung für den DaF-Unterricht haben? In: Andrea Bachmann-Stein, Stephan Stein (Hg.): Mediale Varietäten: Gesprochene und geschriebene Sprache und ihre fremdsprachendidaktischen Potenziale. Landau: Verlag Empirische Pädagogik, 39-61.

Imo, Wolfgang (2011): ,Jetzt gehn wir einen trinken, gell?' Vergewisserungssignale (tag questions) und ihre Relevanz für den DaF-Unterricht. In: Sandro Moraldo (Hg.): Deutsch aktuell 2. Einführung in die Tendenzen der deutschen Gegenwartssprache. Rom: Carocci, 127-150.

Imo, Wolfgang (2012): Hattu Möhrchen? Gesprochene Sprache im DaF-Unterricht. In: Ulrike Reeg, Pasquale Gallo, Sandro M. Moraldo (Hg.): Gesprochene Sprache im DaF-Unterricht: Zur Theorie und Praxis eines Lerngegenstandes. Münster: Waxmann, 29-56.

Imo, Wolfgang (2013a): Sprache-in-Interaktion: Analysemethoden und Untersuchungsfelder. Berlin: de Gruyter.

Imo, Wolfgang (2013b): , Rede und ,Schreibe': Warum es sinnvoll ist, im DaF-Unterricht beides zu vermitteln. In: Sandro M. Moraldo, Federica Missaglia (Hg.): Gesprochene Sprache im DaF-Unterricht. Heidelberg: Winter, 59-82.

Imo, Wolfgang (2015): Aspektrealisierung im gesprochenen Deutsch zwischen Norm und Gebrauch. In: Sandro M. Moraldo, Wolfgang Imo (Hg.): Interaktionale Sprache im DaF-Unterricht. Tübingen: Stauffenburg, 367-393.

Imo, Wolfgang / Lanwer, Jens P. (2019): Interaktionale Linguistik. Eine Einführung. Stuttgart: Metzler. Imo, Wolfgang / Moraldo Sandro M. (Hg.) (2015): Interaktionale Sprache und ibre Didaktisierung im DaF-Unterricht. Tübingen: Stauffenburg.

Kilian, Jörg (2005): DaF im Chat. Zur Grammatik geschriebener Umgangssprachen als Ergänzung zum Erwerb standardsprachlichen Wissens. In: Michael Beißwenger, Angelika Storrer (Hg.): Chat-Kommunikation in Beruf, Bildung und Medien: Konzepte - Werkzeuge - Anwendungsfelder. Stuttgart: ibidem, 201-220.

Kotin, Michail L. (2019): Grammatikforschung und Grammatikschreibug: Zur Überbrückung der Kluft zwischen Forschung und Lehre im universitären Grammatikunterricht. In: Studia Niemcoznawcze LXIII, 45-56.

Lüger, Heinz-Helmut (1991): Indirektheit und Höflichkeit - ein landeskundliches Thema? In: Beiträge zur Fremdsprachenvermittlung 22, 93-115. 
Lüger, Heinz-Helmut (1993): Höflichkeit und Lehrbuchdialog. In: Heinrich Löffler (Hg.): Dialoganalyse $I V$, Teil I. Tübingen: Niemeyer, 233-240.

Lüger, Heinz-Helmut (1995a): Gesprächsanalyse und Fremdsprachenvermittlung. In: Heinz-Helmut Lüger (Hg.): Beiträge zur Fremdsprachenvermittlung, Gesprächsanalyse und Gesprächsschulung. Konstanz: SLI, 3-21.

Lüger, Heinz-Helmut (1995b): Partnerorientiertes Sprechen in Lehrbuchdialogen. In: Heinz-Helmut Lüger (Hg.): Beiträge zur Fremdsprachenvermittlung, Gesprächsanalyse und Gesprächsschulung. Konstanz: SLI, 111-123.

Lüger, Heinz-Helmut (2009): Authentische Mündlichkeit im fremdsprachlichen Unterricht?. In: Andrea Bachmann-Stein, Stephan Stein (Hg.): Mediale Varietäten. Gesprochene und geschriebene Sprache und ibre fremdsprachendidaktische Potenziale. Landau: Empirische Pädagogik, 15-37.

Moraldo, Sandro M. (2012): Korrektivsätze (obwohl, obgleich, obschon, obzwar) - Zur Grammatik korrektiven Konnektoren und ihrer Bedeutung für den interkulturellen Fremdsprachenunterricht. In: Ulrike Reeg, Pasquale Gallo, Sandro M. Moraldo (Hg.): Gesprochene Sprache im DaF-Unterricht. Zur Theorie und Praxis eines Lerngegenstandes. Münster u. a.: Waxmann, 99-120.

Moraldo, Sandro M. (2013): «Ich muss Kunst und Deutsch lernen. Obwohl- nee, Deutsch lernen hab ich nicht nötig Sprachwandel und Sprachvariation: obwobl-Sätze im DaF-Unterricht. In: Sandro M. Moraldo, Federica Missaglia (Hg.): Gesprochene Sprache im DaF-Unterricht. Grundlagen Ansätze - Praxis, Heidelberg: Winter, 267-281.

Moraldo, Sandro M. / Missaglia, Frederica (Hg.) (2013): Gesprochene Sprache im DaF-Unterricht. Grundlagen - Ansätze - Praxis, Heidelberg: Winter.

Neuland, Eva (Hg.) (2006): Variation im heutigen Deutsch. Perspektiven für den Sprachunterricht. Frankfurt/M.: Lang.

Neuland, Eva (2011): Variation in der deutschen Sprache und ihre Auswirkungen auf den (Fremd) Sprach(en)unterricht. In: Sandro Moraldo (Hg.): Deutsch aktuell 2. Tendenzen der deutschen Gegenwartssprache. Rom: Carocci, 48-63

Neuland, Eva (2013): Gesprächsmuster und Variationen der mündlichen Kommunikation im DaFUnterricht. In: Sandro M. Moraldo, Federica Missaglia (Hg.): Gesprochene Sprache im DaF-Unterricht. Grundlagen - Ansätze - Praxis. Heidelberg: Winter, 151-169.

Neuland, Eva (2018): Aktuelle Sprachwandelprozesse als Gegenstand der Reflexion im DaF-Unterricht. In: Sandro M. Moraldo (Hg.): Sprachwandel. Perspektiven für den Unterricht Deutsch als Fremdsprache. Heidelberg: Winter, 29-48.

Pieklarz-Thien, Magdalena (2015): Gesprochene Sprache in der philologischen Sprachausbildung. Theoretische Grundlagen - empirische Befunde - exemplarische Anwendungen. Frankfurt/M.: Lang.

Pieklarz-Thien, Magdalena (2018a): Von der disziplinären Abhängigkeit zum gleichberechtigten Miteinander? Eine Diskussion über das sensible Verhältnis von Sprachwissenschaft und Sprachdidaktik am Beispiel der Germanistischen Linguistik und der DaF-Didaktik. In: Glottodidactica XLV/1, $115-130$.

Pieklarz-Thien, Magdalena (2018b): Standardowa niemczyzna mówiona w kształceniu językowym na poziomie filologicznym. Krótkie podsumowanie najważniejszych wniosków z badań własnych i prezentacja przykładowych rozwiązań praktycznych [Gesprochenes Standarddeutsch in der philologischen Sprachausbildung. Kurze Darstellung der Ergebnisse und Folgerungen einer empirischen 
Untersuchung und Präsentation der exemplarischen Anwendungen]. In: Prace Jezzykoznawcze 20/4, 207-226.

Pieklarz-Thien, Magdalena (2020a): Sprache als Forschungs- und Lehr-/Lerngegenstand. Eine Diskussion über die Divergenz zwischen der linguistischen und didaktischen Sprachauffassung und ihre Konsequenzen im germanistischen Deutschunterricht im Ausland. In: Deutsch als Fremdsprache. Zeitschrift zur Theorie und Praxis des Faches Deutsch als Fremdsprache (im Druck).

Pieklarz-Thien, Magdalena (2020b): Gesprochenes Standarddeutsch - Annahmen und Grundlagen zur Vermittlung eines komplexen sprachlichen Lerngegenstandes im germanistischen DaF-Unterricht in Polen. In: Susanne Günthner u. a. (Hg.): Gesprochene Sprache in der kommunikativen Praxis Analysen authentischer Alltagssprache und ihr Einsatz im DaF-Unterricht. Tübingen: Stauffenburg (im Druck).

Prokop, Izabela (Hg.) (1990): Gesprochene Sprache I. Materialien des I. wissenschaftlichen Symposiums im Rahmen des Forschungsprojekts ,Linguistische Studien zur gesprochenen Sprache‘, Poznań, 23.-29.06.1988. Poznań: Wydawnictwo UAM.

Reeg, Ulrike / Gallo, Pasquale / Moraldo, Sandro M. (Hg.) (2012): Gesprochene Sprache im DaFUnterricht. Zur Theorie und Praxis eines Lerngegenstandes. Münster u. a.: Waxmann.

Reershemius, Gertrud (1998): Gesprochene Sprache als Gegenstand des Grammatikunterrichts. In: Info DaF 25/4, 399-405.

Richter, Regina (2002): Zur Relevanz der Gesprochene-Sprache-Forschung für den DaF-Unterricht. In: Info DaF 29/4, 306-316.

Rothstein, Björn / Schmadel, Saskia / Wöllstein, Angelika (2014): Bessere Grammatische Kenntnisse des Deutschen durch zusätzlichen Grammatikunterricht in der Sekundarstufe II? Das Projekt GramKidSII. In: Mitteilungen des Deutschen Germanistenverbandes 2, 203-207.

Schatte, Czesława (1993): Probleme der Vermittlung der gesprochenen Sprache im Fremdsprachenunterricht. In: Günther Richter (Hg.): Methodische Grundfragen der Erforschung gesprochener Sprache. Frankfurt/M.: Lang, 135-142.

Schilling, Andrea / Stezano Cotelo, Kristin (2008): Wie komm ich zu Wort? Redewechsel als Thema im DaF-Unterricht. In: Christoph Chlosta, Gabriela Leder, Barbara Krischer (Hg.): Auf neuen Wegen. Deutsch als Fremdsprache in Forschung und Praxis. 35. Jahrestagung des Fachverbands Deutsch als Fremdsprache an der Freien Universität Berlin 2007. Materialien DaF, Band 79. Göttingen: Universitätsverlag, 323-342.

Schneider, Jan G. / Butterworth, Judith / Hahn, Nadine (Hg.) (2018): Gesprochener Standard in syntaktischer Perspektive. Theoretische Grundlagen - Empirie - didaktische Konsequenzen. Tübingen: Stauffenburg.

Schwitalla, Johannes (1997/42012): Gesprochenes Deutsch. Eine Einführung. Berlin: Schmidt.

Sieberg, Bernd (2013): Sprechen lehren, lernen und verstehen. Grammatik und Übungen zu Ausdrucksweisen und Strukturen mündlichen Kommunikation. Stufenübergreifendes Studien- und Übungsbuch für den DaF-Bereich. Tübingen: Julius Groos.

Spiekermann, Helmut (2007): Standardsprache im DaF-Unterricht: Normstandard - nationale Standardvarietäten - regionale Standardvarietäten. In: Linguistik online 32/3, 119-137.

Thurmair, Maria (2002): Standardnorm und Abweichungen. Entwicklungstendenzen unter dem Einfluss der gesprochenen Sprache. In: Deutsch als Fremdsprache. Zeitschrift zur Theorie und Praxis des Deutschunterrichts für Ausländer 39, 3-9. 
Thurmair, Maria (2005): < Aber man spricht doch ganz anders heute!?> Wortstellungsvariationen der gesprochenen Sprache im Unterricht Deutsch als Fremdsprache. In: Deutsch als Fremdsprache $32,42-48$.

Thurmair, Maria (2013): Gesprochene Sprache und DaF. In: Bernt Ahrenholz, Ingelore Oomen-Welke (Hg.): Deutsch als Fremdsprache. Deutschunterricht in Theorie und Praxis. Baltmannsweiler: Schneider Verlag Hohengehren, 98-106.

Thurmair, Maria (2018): An der Schnittstelle von DaF und Germanistischer Sprachwissenschaft. Bestandsaufnahme und Perspektiven. In: Angelika Wöllstein u. a. (Hg.): Grammatiktheorie und Empirie in der Germanistischen Sprachwissenschaft (FS Ludwig M. Eichinger). Berlin / Boston: de Gruyter, 409-432.

Tomczyk-Popińska, Ewa (1994): Dialogi w podręcznikach języka niemieckiego [Dialoge in den DaF-Lehrwerken]. In: Przeglad Glottodydaktyczny 13, 47-54.

Tomczyk-Popińska, Ewa (1997): Untersuchungen zu mediolektalen Unterschieden im dialogischen Gegenwartsdeutsch. Didaktische Implikationen.Warszawa: SCRIPT.

Tomczyk-Popińska, Ewa (2003): Zróżnicowanie geograficzne języka niemieckiego w nauczaniu tego języka w Polsce - wybrane aspekty [Geographische Variation der deutschen Sprache im DaFUnterricht in Polen - ausgewählte Probleme]. In: Przeglad Glottodydaktyczny 19, 47-51.

Tomczyk-Popińska, Ewa (2006): Nauczanie porozumiewania się w zróżnicowanych sytuacjach komunikacyjnych - wnioski z analiz podręczników języka niemieckiego jako obcego [Die Verständigung in vielfältigen Kommunikationssituationen als Unterrichtsgegenstand - Schlussfolgerungen aus der Analyse der DaF-Lehrwerke]. In: Przeglad Glottodydaktyczny 19, 67-75.

Vorderwülbecke, Klaus (2008): Sprache kommt von Sprechen - Gesprochene Sprache im DaFUnterricht. In: Christoph Chlosta / Gabriela Leder / Barbara Krischer (Hg.): Auf neuen Wegen. Deutsch als Fremdsprache in Forschung und Praxis. 35. Jahrestagung des Fachverbands Deutsch als Fremdsprache an der Freien Universität Berlin 2007. Materialien DaF, Band 79. Göttingen: FaDaF, 275-292.

Wawrzyniak, Zdzisław (1971): Gesprochenes Deutsch. Skrypt dla studentów germanistyki. Kraków: Uniwersytet Jagielloński.

Weinrich, Harald (42007): Textgrammatik der deutschen Sprache. Hildesheim: Olms.

Zifonun, Gisela / Hoffmann, Ludger / Strecker, Bruno (1997): Grammatik der deutschen Sprache. Band 1-3. Berlin / New York: de Gruyter. 